instruments, the Observatory being equipped at present with two horizontal Milne-Shaw seismographs. The two earth tremors felt at Hemsworth and South Elmsall in south Yorkshire on October 23, 1954 (see Nature, 175, 587; 1955), have also been investigated by Mr. Baxter. The first was recorded at Durham, at Kew and at Rathfarmham near Dublin, though not at Edinburgh. From an analysis of the seismograms, Mr. Baxter has determined the epicentre at lat. $53^{\circ} 36^{\prime} \mathrm{N}$., long. $01^{\circ} 19^{\prime} \mathrm{W}$., and time of origin 13h. $26 \mathrm{~m} .10 \mathrm{~s}$. G.M.T. The strength of the shock macroseismically was III on the Modified Mercalli scale.

At the National Institute of Oceanography, Mr. J. Darbyshire has calculated refraction diagrams for microseismic waves approaching Bermuda on the assumption that they are surface waves between the ocean and the sea-bed of the type described by Stoneley. The diagrams show that the refraction of energy away from the island is very marked for waves approaching from the north-east and southwest. This effect explains some of the anomalies observed at Bermuda, in one of which a storm slightly to the west of north gave large microseisms, whereas one slightly to the east of north had little effect. The work shows that the study of recordings at islands must take account of refraction.

International co-operation is most important in seismology as in other sciences and, apart from private research and personal contacts, British seismologists have been recently active with the loan of instruments (a new driving clock has been ordered for the instrument at Suva, Fiji, with money from the Gray-Milne Fund), through the International Seismological Summary, and through attendance at the tenth General Assembly of the International Union of Geodesy and Geophysics in Rome during September 14-25, 1954. The International Seismological Summary is under the direction of Sir Harold Jeffreys, the calculations being done at Kew by Mr. J. S. Hughes and his staff. The work for June 1947 is in hand, the first five months of the year being ready for printing. The third quarter for 1946 will be distributed shortly. Dr. Stoneley reports that the programme of the General Assembly of the International Union of Geodesy and Geophysics was a very full one and was notable for the number of interesting and successful symposia and joint meetings of associations. For the first time since the Second World War, Soviet geophysicists were present, attending by invitation ; in particular, the names of Prof. Vladimir Beloussov, of the Moscow Academy of Sciences, and Prof. Georgy Gorshkov, of the Moscow State University, may be mentioned. The Assembly was preceded by a reunion of the European Seismological Commission, under the presidency of Prof. W. Hiller. The proceedings of the Association of Seismology and the Physics of the Earth's Interior opened with a presidential address by Prof. B. Gutenberg on "The Effects of Low-Velocity Layers", and some of the joint meetings held were as follows: constitution of the Earth and dissipation of energy (with vulcanology); isostasy (with geodesy), and gravimetric and seismic interpretation; variation of latitude (with geodesy and vulcanology). In addition to special sessions on apparatus, on microseisms and on seismography, the grouping of papers under headings gave the meetings more of the character of symposia. The considerable development of seismology during recent years was evident.

ERnest TIlLotson

\section{ADVANCES IN MOLECULAR SPECTROSCOPY}

\author{
MEETING IN OXFORD
}

7 HE European Molecular Spectroscopy Group, which was constituted informally after the Second World War and held meetings in Constance (1947), Basle (1951) and Paris (1953), met again this year, in Oxford, during July 7-11. This meeting was a great success, and thanks are due to the industrial firms whose financial help made it possible. Among two hundred and eighty participants were representatives from fourteen European countries, Brazil, the United States, Canada, South Africa and Japan. More than eighty papers were presented, covering many topics in ultra-violet, infra-red and Raman spectroscopy, with an emphasis on spectral theory and the correlation of spectra with molecular constitution and reactivity rather than on technique or analytical applications.

The ease with which spectra can now be photographed or recorded automatically has stimulated more attention to different conditions of excitation and to the physico-chemical factors which determine detailed spectral characteristics. For example, very interesting results are emerging from the study of infra-red absorption spectra of gaseous mixtures at high pressure, or of mixtures of liquids. B. Vodar (Bellevue, Paris) and his colleagues, using mixtures of hydrogen chloride and hydrogen at high pressure, have found an infra-red absorption band corresponding to $\left(\nu_{\mathrm{HCl}}+\nu_{\mathrm{H}_{2}}\right)$, the combination of the active hydrogen chloride fundamental with the inactive vibration of hydrogen. Also, the absorption band due to $v_{H}$, which becomes active at high pressures, is affected by hydrogen chloride or other added gases. Similarly, J. Fahrenfort (University of Amsterdam) has found that using compressed mixtures of carbon dioxide with nitrogen, oxygen or hydrogen, absorption bands are observed due to simultaneous transitions in a collision pair, such as $\left(\nu_{\mathrm{CO}_{2}}+\nu_{\mathrm{N}_{2}}\right)$ or $\left(\nu_{\mathrm{CO}_{2}}+y_{\mathrm{H}}\right)$; he has examined this effect in terms of the mutual molecular polarization and has found satisfactory agreement between the predicted and observed band intensities. All these measurements bear on the question of the formation of transient molecular complexes or aggregates, quite apart from the general information they may provide about intermolecular forces.

Analogous effects have been found for liquid mixtures by J. Ketelaar (University of Amsterdam). In thick layers, a carbon disulphide-bromine mixture shows bands at $1,807 \mathrm{~cm} .^{-1}$ and $1,204 \mathrm{~cm} \cdot .^{-1}$, lying respectively $306 \mathrm{~cm}^{-1}$ on either side of the strong absorption band of carbon disulphide at $1,510 \mathrm{~cm} .^{-1}$. This displacement interval corresponds to the vibration frequency of bromine. When polyatomic molecules are mixed with carbon disulphide, an array of displaced bands is found, analogous to a vibrational Raman spectrum spread around the main carbon disulphide band at 1,510 $\mathrm{cm}^{-1}$. Thus we may not only detect collision complexes but also determine the low frequencies of a heavy molecule in a more convenient spectral region. H. L. Welsh (University of Toronto) described further work on the 'forbidden' infra-red bands of carbon dioxide and hydrogen in the liquid and solid states. At low temperatures these bands assume a more complex pattern in consequence of intermolecular inter- 
actions and specific properties of the crystalline solids, and combination transitions of hydrogendeuterium molecular complexes have also been observed.

The interpretation of vibrational band intensities, which are as significant as the frequencies, has progressed rapidly. These intensities are controlled by the polar characteristics of the vibrations concerned, and therefore lead to deductions about bond polar properties and the electronic structure of the whole molecule. Since, moreover, the creation during molecular vibration of centres of residual electrical charge may be a prelude to reactivity, the absorption band intensitios may be correlated with the latter. True integrated absorption band intensities, as opposed to their 'apparent' values or the 'apparent' extinction coefficients, can now be determined with fair accuracy. Use of these data is limited, however, by uncertainties in the molecular force fields and by ambiguities which arise in part since the square root of the intensity has an indeterminate sign. One possible way out is to study a set of isotopic molecules where for a given vibration-type the derivative o electric moment with respect to a symmetry coordinate should be constant. B. L. Crawford (University of Minnesota) described such work on ethylene and the deutero-ethylenes, and on the methyl halides, D. F. Hornig (Brown University) on methyl cyanide and the cyanogen halides, and D. H. Whiffen (University of Birmingham) on benzene. It is noteworthy that in some of these structures, carboncarbon bonds are found to have fairly large dipole moments.

S. Silverman (Johns Hopkins University) has determined the integrated band intensities of hydrogen halides and carbon monoxide from measurements on individual rotation lines, and has analysed the results in terms of a new more detailed theory which assumes a more general expression for the variation of dipole moment with bond-length and which also considers vibration-rotation interaction. Theoretical work on the band intensities of hydrogen halides was also discussed by S. Penner (California Institute of Technology, Pasadena).

The function expressing variation of a bond dipole with change in length, $M=M_{0}+M_{1} \xi+M_{2} \xi^{2}+\ldots$, has assumed greater interest since it is now realized that most vibrations involve considerable electrical anharmonicity, expressed by the quadratic and higher terms in $\xi$, and this determines the overtone intensities more than mechanical anharmonicity. H. W. Thompson and D. W. Russell (Physical Chemistry Laboratory, Oxford) described work on the bands $v_{N H}$ and $2 v_{\mathrm{NH}}$ of a selected series of complex organic molecules including aliphatic and aromatic secondary amines, amides and heterocyclic bases, from which the coefficients $M_{1}, M_{2}$, can be derived and correlated with variations of ionic character in the different kinds of NH bond.

Attempts have been made to correlate the variation of vibration frequency of a key group in a related series of molecules with changes in reactivity. L. J. Bellamy (Waltham Abbey) discussed further examples of substituted benzenes in which a relation exists between a particular vibration frequency and the Hammett $\sigma$-factor for the particular substituent. Such relations might be applied to estimate dissociation constants of acids and bases, redox potentials, etc., although there are some anomalies and the frequency variations are often small. Any inductive or mesomeric effects will be similarly reflected in the bond polar properties, and the consequential variation of band intensities may be more pronounced than the frequency shifts. Thus, H. W. Thompson showed that for the $\mathrm{C} \equiv \mathrm{N}$ bond vibration in substituted benzonitriles there is a proportionality between log (intensity) and $\sigma$ over a wide range, and similar correlations between the intensity and several reaction constants. A striking difference of intensity of $v_{C N}$ in the solvent carbon tetrachloride or chloroform was noticed in cases where hydrogen bonding of chloroform to the solute could occur favourably, which emphasizes the need for care where quantitative analysis is being carried out in apparently innocuous solvents. The intensities of NH-group vibration bands in platinum-amine complexes have also been correlated with electronic structure by L. A. Duncanson (Imperial Chemical Industries, Ltd., Welwyn); and G. Higgins (British Rubber Producers' Research Association, Welwyn) described how infrared studies of the association equilibria between organic hydroperoxides, phenols or alcohols with dialkyl sulphoxides may be used to estimate the dissociation constants of weak acids.

Higher resolving-power in the infra-red, obtained with better detectors and gratings, is being used to obtain further details of molecular dynamics. Fermi resonance between vibrational levels often leads to anomalous values in the coefficients $\alpha_{i}$ relating the rotational constants in different vibrational states, and also in the centrifugal stretching-constants $D_{i}$. More accurate values can also be obtained for the Coriolis coupling factors $\zeta_{i}$ and $l$-doubling coefficients in degenerate vibrational levels. Such work on carbon dioxide, nitrous oxide and allene was described by H. H. Nielsen (Ohio State University), on some hydrides of the fourth-group elements by M. Kent Wilson (King's College, London), and on acetylene, methyl acetylene, hydrogen cyanide and their deuterium analogues by $\mathrm{H}$. W. Thompson. A further revision of the bond-lengths in acetylene seems necessary. W. F. Edgell (Purdue University) explained a new method whereby the measurement of vibrational band contours promises to yield values for the molecular rotational constants.

Many papers were concerned with general infrared developments and vibrational analysis. L. Genzel (University of Frankfurt) described a now grating spectrometer for use to $1,000 \mu$, using a high-pressure mercury arc as source with suitable filters. $R$. C. Lord (Massachusetts Institute of Technology) and his colleagues have found from the spectra of cyclo. butane and cyclopropane and of their deutero analogues that the former appears to contain a puckered ring, and in the latter $r_{c c}=1524 \mathrm{~A}$. R. Mecke (University of Freiburg) has measured the spectra of homologous series of $\alpha \omega$-dibromoparaffins and alkyl bromides in the liquid and solid phases. In the solids, rotational isomerism is excluded, and by considering the whole series a reasonable assignment of the carbon-chain vibrations can be made. S. F. D. Orr (Chester Beatty Research Institute, London) has found that in trans stilbenes, although the integrated intensity of the nonplanar $=\mathrm{C}-\mathrm{H}$ deformation is unaffected by ortho substituents, the band-width and peak extinction coefficients are much affected, and an explanation in terms of vibrational damping is suggested. N. Sheppard (Laboratory of Colloid Science, Cambridge) has assigned the vibration frequencies of the guanidinium ion, $\mathrm{C}\left(\mathrm{NH}_{2}\right)+_{3}$, in crystals and solid films, and data relevant to the study of ureas and amides. Mlle. M. L. Josien 
(University of Bordeaux) and colleagues have measured $v_{00}$ in ketones and in quinones of mono. and poly-nuclear aromatics, while N. Fuson (Fisk University) has studied substituted benzanthracenes of carcinogenic interest. The assignment of $\mathrm{OH}$-group vibrations in enolized diketones and ketonic alcohols has been explored by D. Hadzi (University of Ljubljana) and by C. Duculot (University of Paris), and W. Lüttke (University of Freiburg) has studied the dimerization of nitroso compounds.

A packed session of the meeting, on the infra-red spectra of amides, peptides and polymers, served to emphasize the difficult stage which this work has reached, and the hazards of interpreting spectra with polarized radiation. G. B. B. M. Sutherland (University of Michigan) suggested that analysis in terms of group frequencies can only provide a partial interpretation. With crystalline polymers, in which a structural unit is visualized in the unit cell, appropriately influenced by its environment, more exact selection rules will apply than with liquid or amorphous polymers. He illustrated this argument by reference to polyethylene, ice and other substances. From results with crystals of naphthalene, iodoform and thiourea, R. M. Hexter (Cornell University) claimed to verify a point made several years ago, but not sufficiently appreciated, that transition moments of vibrations are often not parallel or perpendicular to bond directions; and he suggested that if dichroic ratios are to be correctly determined the complete anisotropy must be measured. A. Elliott (Courtaulds, Ltd.) has found that with crystals of acetanilide the transition moment of the carbonyl group is inclined to the bond direction.

E. K. Blout (Polaroid Corporation) observed that, whereas lower members of a series of polybenzyl glutamates have $v_{\mathrm{CO} O}=1,628 \mathrm{~cm}^{-1}$, the higher members have $v_{\mathrm{CO}}=1,655 \mathrm{~cm}^{-1}$, in line with $\beta$ - and $\alpha$-configurations of polypeptides; and he suggested that some previous discrepancies between different laboratories might arise from degradation of polymers during the casting of films from solvents. H. A. Willis and R. G. J. Miller (Plastics Division, Imperial Chemical Industries, Ltd.) proposed a method for determining the crystalline-amorphous content of polymers such as polyethylene or polyethylene terephthalate, based only upon the broad bands of the amorphous form which are associated with the multiplicity of rotational isomers; J. Mann and J. Marrinan (British Rayon Research Association) showed how the hydroxyl group bands in different crystalline forms of cellulose can be used to charac. terize the latter; the old problem of the 'amide group' bands in the gaseous, liquid and crystalline state was reconsidered by S. Mizushima (University of Tokyo) and by M. Davies (Aberystwyth); and D. S. Barmby (Wool Industries Research Association, Leeds) ontlined the effect on key bands of nylon when it is dissolved in antimony trichloride-a possible start to the study of the swelling of polymers.

As regards theoretical aspects, C. A. Coulson (Mathematical Institute, Oxford) showed how data on the lengths of carbon-carbon bonds can now be systematized in terms of specific types of hybridization and hyperconjugation. J. W. Linnett (Inorganic Chemistry Laboratory, Oxford), in reviewing possible ways of assessing molecular force fields, suggested that more use might be made of isotopic effects other than with hydrogen-deuterium, and that the Coriolis factors $\zeta_{i}$ and centrifugal stretching constants $D^{i}$ mentioned previously may also be useful. New methods were outlined by P. W. Higgs (Mathematical Physics Department, Edinburgh) for calculating the vibration frequencies of complex molecules, and by H. Günthard (Federal Technical Institute, Zuricl) for obtaining relations between the intensities of bands of isotopic molecules, while G. Amat (P.C.B., Paris) considered the effects of Fermi resonance upon molecular rotational and vibrational energy-levels in detail. P. Tuomikowski (University of Helsinki) described comparative studies of Fermi resonance for a given molecule in different states of aggregation.

Raman spectra of coloured substances, using red or near infra-red radiation as the exciting source, were described by H. Stammreich (University of São Paulo); discharge lamps of helium, rubidium or crsium were used, and the substances studied included bromine, iodine chloride, iodoform and polyhalide ions. Raman spectroscopy of gases with high resolution, using multi-reflexion cells and highcurrent water-cooled mercury arcs, is advancing rapidly, and H. L. Wolsh (University of Toronto) gave new data on vibration-rotation bands of a number of simple hydrocarbons. This method not only provides information about certain bands not obtainable in the infra-red, but also the confusing influence of 'hot' bands is sometimes minimized. Several speakers discussed Raman spectra of crystals and complex ions, and on the basis of intensities of Raman bands in homologous series of ketonas, esters and nitro compounds G. Michel (University of Liège) suggested that rules can be set up for analysis and structural diagnosis. D. A. Long (Physical Chemistry Laboratory, Oxford) discussed the more fundamental relationships between Raman band intensities and bond polarizabilities, with special reference to tetrahalides and related structures. Progress here runs parallel to that already mentioned in which infra-red band intensities are being correlated with bond polar properties.

W. C. Price (King's College, London), B. Vodar (Bellevue, Paris) and A. D. Walsh (University of Leeds) discussed technical advances in the vacuum ultra-violet and the correlation of absorption spectra with molecular ionization potentials. Improved emission sources and continuous photoelectric recording should lead to further progress in this field. $H$. Schüler (Hechingen) has obtained evidence for the ultra.violet emission spectra of benzyl and benzal radicals produced in the positive column of a glow discharge in vapours of certain benzene derivativesan experimental method which may provide many data on the emission spectra of polyatomic molecules. The absorption spectra of such radicals have also been measured by G. Porter (British Rayon Research Association) after 'trapping' radicals formod by flash photolysis in a rigid medium where they persist for long periods in metastable equilibrium. This trick of retaining simple polyatomic radicals in solid matrices was also described by G. Pimentel (University of California). The use of crystals of aromatic compounds at very low temperatures has also enabled P. Pesteil (Sorbonne, Paris) to obtain sharper fluorescence spectra $(T \rightarrow S)$ and to determine many molecular vibrational levels. Other ultra-violet studies included the exciton spectra in inorganic crystals at low temperature (S. Nikitine, University of Strasbourg); the effect of substituents on the spectra of diphenyls and benzophenones (G. H. Beaven, National Institute for Medical Research, Mill Hill, and R. F. Rekker, Free University of Amsterdam); the spectra of benziminazoles and 
thiophenes; and many new spectra of diatomic oxides, halides and diatomic ions which extend general theories of molecular structures further. H. C. Longuet-Higgins (Chemistry Laboratory, Cambridge) analysed theoretically the electron states of a composite system in relation to those of its constituent parts and showed how some general ideas about electron transfer configurations can be used in this connexion. R. S. Mulliken (University of Chicago) and C. J. Timmons (University of Nottingham) discussed charge transfer and charge resonance spectra and the correlation of spectra with intermolecular complex formation.

\section{H. W. Thompson}

\section{PROGRESS IN RADIOBIOLOGY}

\section{CONFERENCE AT CAMBRIDGE}

$\mathrm{O}$ $\mathrm{N}$ August 15, radiobiologists met in Cambridge for the third annual conference to discuss progress in their field. The three-day meeting, under the chairmanship of Sir Ernest Rock-Carling, was organized by Prof. J. S. Mitchell and his staff. Some 250 scientists, from sixteen countries including the U.S.S.R., enjoyed the hospitality of our Cambridge hosts. Eighty papers were discussed, the variety of the contributions reflecting the large number of disciplines on which an understanding of the biological effects of radiation must rest.

Biological lesions due to ionizing radiations must originate in the process of absorption of primary energy. Our current picture of the particle track, and relevant knowledge of the nature of the chemical intermediates, were presented by Prof. F. S. Dainton in an introductory lecture. A number of metabolic steps occur between the initial chemical disturbance and the appearance of damage in the organism, and some papers dealt with such effects. In the bacterium Achromobacter fischeri, the enzymatically controlled luminescence decreases during exposure to X-rays and recovers rapidly afterwards. Both decrease and increase follow an exponential time relationship (O. Hug and I. Wolf). Bacterial enzymes show a lower sensitivity to $\mathrm{X}$-rays at low temperatures when exposed in the dry state or in solution, or when in the intact cell, suggesting some common physicochemical mechanism of inactivation (H. Pauly and B. Rajewsky). It appears, however, that in the etiology of the opacity of rabbit lens caused by $X$-rays, biochemical changes in the lens are secondary to cellular damage in the dividing cells of the lens epithelium (A. Pirie).

The genetic effects of radiation took a prominent place in the discussions. Nucleic acids appear to be the main carriers of hereditary factors, and some insight into the action of radiations on genetic material may be expected from the study of radiation effects on them and their metabolism. Some questions discussed were the effect of ultra-violet light on ribonucleic acid metabolism in starfish oocytes (M. Errera and A. Ficq), the inhibition of deoxyribonucleic acid (DNA) synthesis by $\mathrm{X}$-rays in the early stages of regeneration of rat liver (L. K. Mee), and the interesting results obtained in the irradiation of dry preparations of herring sperm deoxyribonucleic acid (P. A. Alexander and $K$. A. Stacey). The sensitivity of female germ cells of mice to the induction by $\mathrm{X}$-rays of dominant lethal mutations is some seventy times greater at first meiotic metaphase than at a stage seven hours earlier (L. B. Russell and W. L. Russell). In Habrobracon eggs irradiated with ultra-violet light, damage to the nucleus is qualitatively and quantitatively different from cytoplasmic damage (R. C. von Borstel and H. Moser). A surprising result was obtained when a breeding population of Drosophila was exposed to $5 \cdot 1 \mathrm{r}$. per $\mathrm{hr}$. for about five years. Its genetic fitness was only $1-5$ per cent lower than that of an unirradiated population, despite an accumulated dose of 250,000 r. (B. Wallace). We are not justified, however, in extrapolating from insects to mammals on the basis of these data (W. L. Russell). Bacteria and bacteriophage have been used as a simplified model for the study of genetic changes in populations (H. Marcovitch).

Since certain chemicals and $X$-rays cause similar biological damage, it is of interest to study the mechanisms by which these lesions arise. A wholebody dose of $100 \mathrm{r}$. of X-rays, or $1 \mathrm{mgm}$. per $\mathrm{kgm}$. of nitrogen mustard, produce the same damage in rat lymph nodes; but the treatments have dissimilar actions since they are not interchangeable (C. Biagini). $\mathrm{N}, \mathrm{N}$-dichlorethyl-p-amino-phenyl-butyric acid causes mainly lymphoid, whereas 'Myleran' causes mainly myeloid effects in rat bone marrow. The effect of $X$-rays can be reproduced almost exactly by applying both chemicals at once, suggesting two separate physiological pathways for the developing $X$-ray damage (L. A. Elson, D. A. G. Galton, L. F. Lamerton and M. Till).

Factors influencing the radiosensitivity of biological systems were discussed in almost every session. It has been known for some time that radiation effects are usually greater in the presence than in the absence of free oxygen. Tails of young mice were used to demonstrate this with $\mathrm{X}$ - and neutron-irradiation (P. Howard-Flanders; E. A. Wright). The rapid autoxidation of cysteine in aqueous solutions suggests that a number of protective effects ascribed to this compound could be accounted for by anoxia (L. H. Gray). Cysteine and cysteamine have a stimulating effect on the oxygen consumption of liver slices. Their protective effect may therefore be due to $a$ reduction in the amount of free oxygen in some organs. (D. W. van Bekkum). Chemical protection and anoxia do not, however, always seem to act along identical pathways (R. W. Brauer, J. S. Krebs and R. Pessotti ; J. Maisin, P. Maldagne, A. Dunjic and H. Maisin). Further, cysteine and cysteamine react with the sulphhydryl group of proteins and might thus protect the protein (L. Eldjarn and A. Pihl; R. Koch).

The effects of oxygen on radiosensitivity may br explained in terms of the relative efficiencies of oxidizing and reducing radicals in reacting with organic molecules, although the state of oxidation of the acceptor substances is of importance (A. J. Swallow; M. Ebert and A. Howard; H. Laser).

The radiosensitivity of barley seeds and of starch depends on humidity, and is at a minimum at 20 per cent. It was suggested that the cause is physico. chemical rather than physiological (L. Ehrenberg). The production of chromosome aberrations in Vicia $f a b a$ roots by 8-ethoxycaffeine or X-rays is sensitive to oxygen, but adenosine triphosphate appears to be involved only in the case of the caffeine (B. A. Kihl. man). Other work on Vicia indicates that inhibition of oxidative phosphorylation interferes with the rejoining of broken chromosomes (S. Wolff).

It is well established that shielding of hæmopoietic tissue, or implantation of such tissue after irradiation, 